\title{
Kalkulierbares Risiko durch Chemo in der Schwangerschaft
}

Frauen, die während einer Schwangerschaft die Diagnose Brustkrebs erhalten, können möglicherweise genauso chemotherapeutisch behandelt werden wie erkrankte Frauen, die nicht schwanger sind. In einer aktuellen Beobachtungsstudie wurden keine mit der Chemotherapie assoziierten Schädigungen der Feten festgestellt.

In der europaweiten Studie wurde bei mehr als 400 Frauen während der Schwangerschaft Brustkrebs im Frühstadium diagnostiziert. 197 Patientinnen (48\%) erhielten während der Schwangerschaft eine Chemotherapie mit im Median vier Therapiezyklen. 178 Patientinnen wurden mit einem Anthrazyklin behandelt, 15 mit Cyclophosphamid, Methotrexat und 5-Fluorouracil. 14 Patientinnen erhielten ein Taxan. Bei allen Frauen begann die Chemotherapie erst im zweiten Trimester. Alle Neugeborenen wurden bis vier Wochen postpartal beobachtet.

Die Komplikationsrate bei den Neugeborenen - etwa gemessen am Apgar, an
Blutungsstörungen und Geburtsdefekten - war in der Therapiegruppe nicht höher als in der Vergleichsgruppe. Zudem beeinflusste die Krebstherapie das Geburtsgewicht nicht.

Im Vergleich zur übrigen Bevölkerung, in der die Rate der Frühgeburten zwischen 10 und $15 \%$ beträgt, lag diese Rate in der Studie mit $50 \%$ allerdings deutlich höher. $23 \%$ der Geburten waren noch vor der 35. Gestationswoche. Auch traten mehr Komplikationen unter den Chemotherapierten auf, meist im Zusammenhang mit einer Frühgeburt. Komplikationen wurden bei insgesamt 40 der 386 Neugeborenen registriert, 31 von ihnen in der Chemotherapiegruppe. Dazu zählten Fehlbildungen wie Polydaktylie oder Rektumatresie sowie Geburtskomplikationen.

Die Autoren diskutieren, dass die Entscheidung für den Geburtszeitpunkt oft ohne medizinische Indikation gestellt werde, und betonen, es gebe keinen zwingenden Grund, die Schwangerschaft vor-

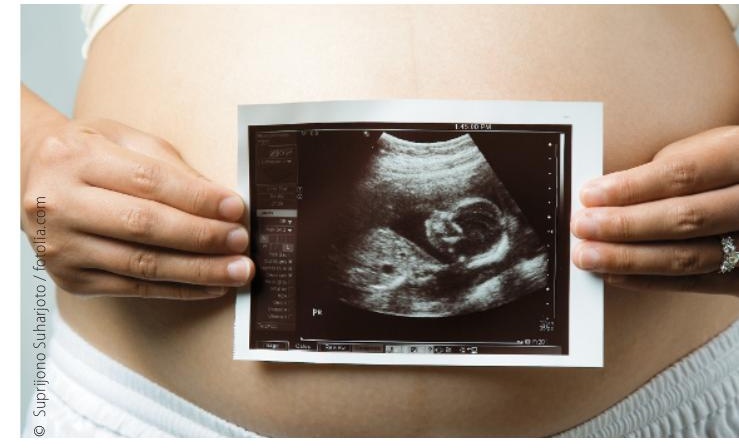

Eine Chemotherapie ab dem zweiten Trimester ist mit dem werdenden Leben vereinbar.

zeitig zu beenden oder eine weniger intensive Chemotherapie als bei nicht schwangeren Patientinnen mit Brustkrebs anzubieten.

Im Kommentar zur Studie betonen Olivier Mir und Paul Berveiller, Paris, dass die optimale Anwendung der zytotoxischen Chemotherapeutika noch offen sei, z. B. die Auswahl der Präparate und deren Dosierung. Auch müsse der langfristige Effekt der Chemotherapie während der Schwangerschaft auf die Entwicklung der Kinder geprüft werden.

Peter Leiner

Loibl S et al. Treatment of breast cancer during pregnancy: an observational study. Lancet Oncol. 2012;13(9):887-96.

\section{Faktoren für ein langes Überleben}

Weltweit ist der Magenkrebs der vierthäufigste maligne Tumor und die zweithäufigste Ursache für einen Krebstod. Auf der Suche nach Faktoren für ein langes Überleben nach Krebsdiagnose sichteten US-Autoren populationsbasierte Daten des California Cancer Registry aus den Jahren 1988 bis 2005.

Von den insgesamt 47.647 im Mittel 70 Jahre alten Patienten mit invasivem Adenokarzinom des Magens und des gastroösophagealen Übergangs verstarben $65,2 \%$ innerhalb von drei Jahren nach Diagnosestellung an ihrer Krebserkrankung. 19,6\% überlebten länger und $15,2 \%$ starben infolge anderer Ursachen oder wurden nicht bis zum Studienende beobachtet. Am häufigsten (39,8\%) befand sich der Tumor am proximalen Ösophagus bzw. an der Kardia. Nur bei
$17,9 \%$ der Patienten war der Tumor zum Zeitpunkt der Diagnose noch lokal, bei $34,5 \%$ bestand eine regionale Ausbreitung und bei $34,8 \%$ hatten sich bereits Fernmetastasen gebildet. Das mittlere krankheitsspezifische Überleben aller Patienten lag bei 11,2 Monaten.

Im Vergleich zu den nach drei Jahren bereits verstorbenen Patienten wiesen die Langzeitüberlebenden häufiger eine Tumorlokalisation in Fundus, Korpus oder Antrum auf. Bei rund $45 \%$ dieser Patienten handelte es sich um einen lokalen Tumor, bei $43 \%$ lag noch eine regionale Begrenzung vor. So konnte sich die Mehrheit der mehr als drei Jahre nach der Magenkrebsdiagnose noch lebenden $\mathrm{Pa}$ tienten (88\%) einer chirurgischen Therapie unterziehen. Ein lokales Tumorwachstum reduzierte die 3-Jahres-Mor- talität um $80 \%$, ein regional begrenztes Stadium um $47 \%$. War die Krankheit 2002 oder später diagnostiziert und der Patient operiert worden, verringerte sich das Risiko insgesamt um $66 \%$, bei Patienten mit lokalem Tumor sogar um $85 \%$. Wurde eine Chemotherapie durchgeführt, stieg die Überlebenschance um $44 \%$, wobei Patienten mit bereits fortgeschrittener Erkrankung die größten Vorteile hatten. Günstig für ein längeres Überleben waren zudem ein Tumor vom intestinalen Typ (HR 0,74 vs. diffuser Typ), eine asiatische Herkunft (HR 0,8 vs. Weiße), die Durchführung einer Strahlentherapie (HR 0,80), ein möglichst hoher Diffenzierungsgrad (HR 0,76) sowie das weibliche Geschlecht (HR 0,92). Auch Art und Größe der Klinik beeinflussten das Ergebnis.

Christine Starostzik

Kunz PL et al. Long-term survivors of gastric cancer: A California population-based study. J Clin Oncology. 2012 Sep 4. [Epub ahead of print]. 